\title{
Measuring NV Centers in Diamond Nanoparticles using Electron Energy Loss Spectroscopy
}

Shery Chang ${ }^{1}$, Haotian Wen ${ }^{2}$, David Kordahl ${ }^{3}$ and Christian Dwyer ${ }^{4}$

${ }^{1}$ Electron Microscope Unit, UNSW, Sydney, New South Wales, Australia, ${ }^{2}$ UNSW, United States, ${ }^{3}$ Centenary College of Louisiana, Shreveport, Louisiana, United States, ${ }^{4}$ Electron Imaging and Spectroscopy Tools, United States

Color centers in diamond are point defects which generally consist of a dopant atom(s) paired with a lattice vacancy (or vacancies). Due to their exceptional photo-stabilities and the biocompatibility of the host, color centers in diamond particles, particularly negatively charged NV centers (NV-), are extensively studied and pursued for applications in quantum sensing, and diagnostic imaging [1]. NV-centers emit visible light that is readily detectable at room temperature, and its spin triplet states enable excellent spin manipulation, showing great potential for many sensing configurations [2].

Compared to other light-emitting nanoparticles, the major drawback in using NV-containing nanodiamonds is their relatively weak fluorescence brightness. Extensive efforts have been made to increase the NV concentration by increasing dopant and/or vacancy concentrations. Modification of the diamond surfaces has also been pursued as a means of stabilizing NV centers. However, it is far from clear which approach is most effective, since the presence of planar defects, strain and surface chemistry/structure all likely influence the vacancy diffusion during NV generation, as well as the charge state of these point defects. Moreover, controlling and monitoring the production of vacancies is extremely challenging, and no established technique currently exists for directly measuring the vacancy concentration in nanocrystalline materials.

Here we demonstrate that it is possible, not only to detect, but to quantify, vacancy-containing point defects in nanodiamond using electron energy loss spectroscopy (EELS) in a TEM. In addition, firstprinciples modelling and electron scattering simulations were used to identify and quantify the associated point defects in core loss and low loss EELS signals.

Figure 1(a) shows the carbon K-edge spectra of nanodiamond particles, along with graphite and bulk diamond reference spectra. In the enlarged view of the near-edge structure (ELNES) in Figure 1(b), a clear peak is present at $282.4 \mathrm{eV}$, as well as features in the range 284-287 eV. Based on the first-principles EELS modeling shown in Figure 1(c), vacancies and NV centers in diamond can be identified by welldefined peaks in the pre-edge of the carbon K-edge spectrum at 282.4 and $282.8 \mathrm{eV}$, respectively. This provides strong evidence that the presence of the experimental pre-peak at $\sim 282.5 \mathrm{eV}$ is associated with vacancies and/or NV centers [3].

While the pre-edge features verified by the first-principles calculations identify the presence of vacancies and/or NV centers, it is difficult to differentiate these two types of point defects using the pre-edge features alone. We have therefore pursued the application of high-energy resolution low-loss EELS to directly identify NV centers [4]. The presence of NV centers in diamonds generate transitions at $\sim 2.1 \mathrm{eV}$ for neutral $\mathrm{NV}\left(\mathrm{NV}^{0}\right)$ and at $\sim 1.9 \mathrm{eV}$ for $\mathrm{NV}^{-}$. We show that it is possible to detect the $\mathrm{NV}$ center using lowloss EELS [4] provided that the diamond surface is relatively pristine. 

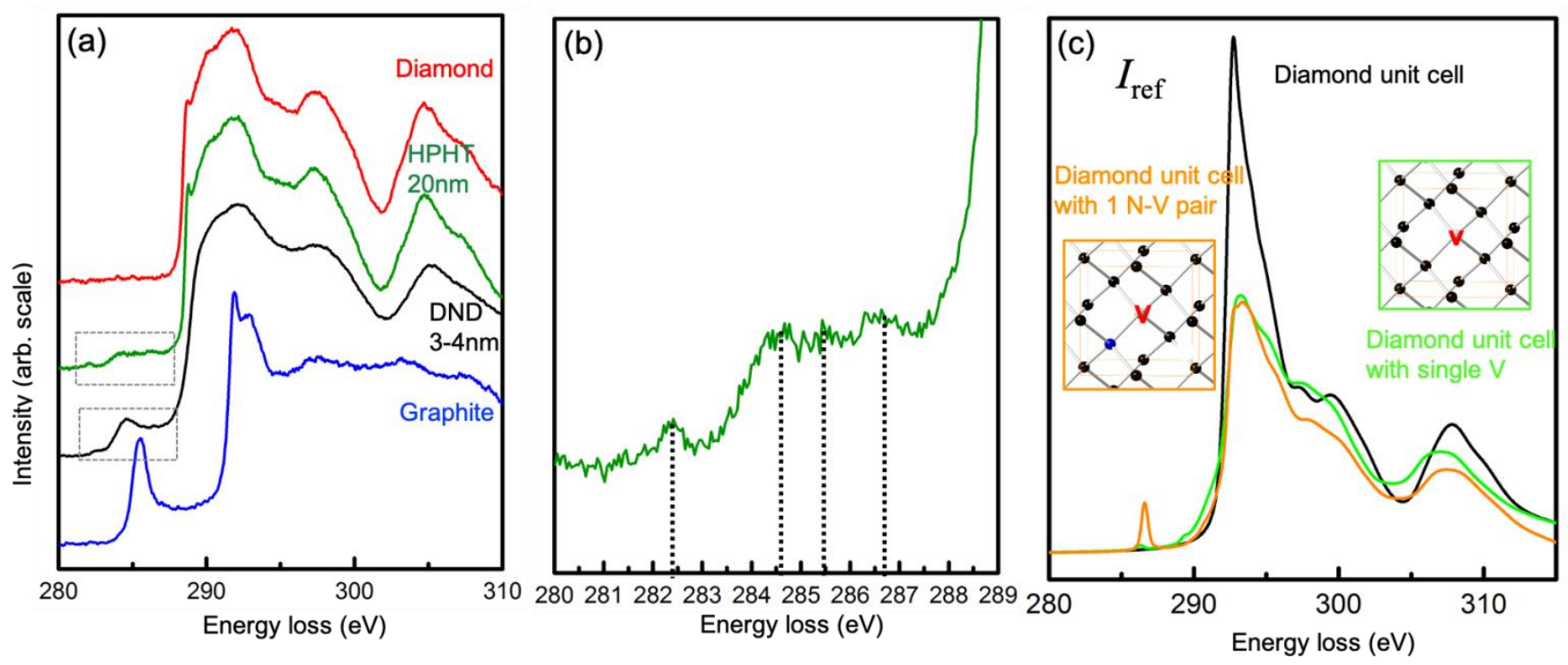

Figure 1. Figure 1: Experimental and calculated carbon K-edge EELS of diamond containing vacancies and NV centers. (a) Spectra of nanodiamond from detonation synthesis (black) and from HPHT process (green), and reference spectra of graphite (blue) and bulk diamond (red); (b) enlarged near-edge structure of HPHT nanodiamond; (c) calculated spectra from pristine diamond (black), and diamond containing an NV center (orange), and isolated vacancy(green) [3].

\section{References}

[1] S Felton et al, Phys. Rev. B 77 (2008) p. 081201.

[2] C Bradac et al, Nature Nanotechnology 5 (2010) p. 345.

[3] S. L Y. Chang et al, Nanoscale (2016).

[4] C. Dwyer et al, (2021) manuscript pending. 